\title{
CARDIAC ARRHYTHMIA PREDICTION BY ADAPTIVE ANALYSIS VIA BLUETOOTH
}

\author{
Ricardo Rodríguez-Jorge ${ }^{1, \bowtie}$, Jiri Bila ${ }^{2}$ \\ ${ }^{1}$ Department of Informatics, Jan Evangelista Purkyně University, Czech Republic \\ ${ }^{2}$ Department of Instrumentation and Control Engineering, Czech Technical University in Prague, Czech Republic \\ ricardo.rodriguezjorge.mx@ieee.org ${ }^{\bowtie}$, Jiri.Bila@cvut.cz
}

\begin{abstract}
In this work, the development of a data acquisition system for adaptive monitoring based on a dynamic quadratic neural unit is presented. Acquisition of the continuous signal is achieved with the BITalino biomedical data acquisition card. The system is trained sample-by-sample with a real time recurrent learning method. Then, possible cardiac arrhythmia is predicted by implementing the adaptive monitoring in real time to recognize patterns that predict cardiac arrhythmia up to 1 second in advance. For the evaluation of the interface, tests are performed using the obtained signal in real time.
\end{abstract}

Keywords: Dynamic quadratic neural unit, recurrent learning, adaptive monitoring, biomedical data acquisition, cardiac arrhythmia, prediction.

Received: 11 November 2020 Accepted: 18 December 2020 Published: 21 December 2020

\section{Introduction}

Cardiovascular disease is a class of diseases that involve the heart or blood vessels, and it can lead to a heart attack, chest pain (angina) or stroke. These diseases remain a serious health problem around the world according to the World Health Organization (WHO). Ischemic heart disease and stroke are examples of cardiovascular diseases, and they were the top major causes of death from 2000 to 2020 [20]. Arrhythmias can be found in individuals with cardiovascular disease (CVD), which is one of the leading causes of death worldwide, representing approximately $27 \%$ of the total deaths from heart disease $[20,26]$.

The electrocardiogram (ECG) is the most common and effective diagnosis tool used to measure and record the electrical activity of the heart in detail [16]. ECG records are obtained by sampling the bioelectric currents sensed by several electrodes, known as leads. The ECG non-stationary signal allows for the detection of cardiac rhythm abnormalities and the diagnosis of a wide range of heart conditions (including arrhythmia) due to it being a non-invasive, effective, simple and inexpensive procedure [1].

An increasing number of people are concerned about their health, and current lifestyle factors are encouraging researchers to develop portable wireless devices capable of monitoring and automatically registering vital signs such as blood pressure, body temperature, electrocardiogram (ECG) signals, and encephalographic signals, among others [26]. An ECG arrhythmia can be defined as any condition in which the electrical activity of the heart is irregular and can cause the heartbeat to become faster or slower; consequently, the blood pressure can be temporally paralyzed and sometimes cause death. Arrhythmias are identified based on where they occur in the heart (atria or ventricles) and based on how the heart's rhythm changes when they occur. Arrhythmias that start in the atria are called atrial or supraventricular (above the ventricles), whereas arrhythmias that start in the ventricles are called ventricular [28].

An arrhythmia may occur at random in the time scale, and symptoms would be manifested at certain irregular intervals during the day but may not appear at all times [1]. An arrhythmia may indicate susceptibility to serious heart disease, stroke or sudden death. Therefore, the early detection and precise discrimination of ECG arrhythmia is essential for patient treatment. The ECG signal has to be observed over several hours to detect a cardiac arrhythmia because the volume of ECG data is enormous. A medical expert may fail to diagnose heart abnormalities due to the nonstationary nature of ECG signals, thereby resulting in life-threatening situations. The analysis of massive ECG data to manually diagnose cardiac disease is very difficult, which makes it a tedious and time consuming task. Therefore, computer-based detection and classification of arrhythmia is one of the critical research fields in clinical cardiology because it can save the life of patients, particularly for the treatment of patients in intensive care units [16].

The proper classification of an ECG signal is achieved by finding the characteristic shapes that can effectively discriminate among required diagnostic categories [29]. The ECG shows each heartbeat as a series of electrical waves. The contractions that pump blood are represented by the $\mathrm{P}$ wave, the QRS complex (union of $\mathrm{Q}, \mathrm{R}, \mathrm{S}$ waves) and $\mathrm{T}$ wave. Occasionally, a U-wave may also be present after the $\mathrm{T}$ wave. The $\mathrm{P}$ wave represents activity in the upper chambers of the heart, whereas the QRS complex and $\mathrm{T}$ wave 
represent activity in the lower chambers [11]. A single cardiac cycle of an ECG is presented in Fig. 1.

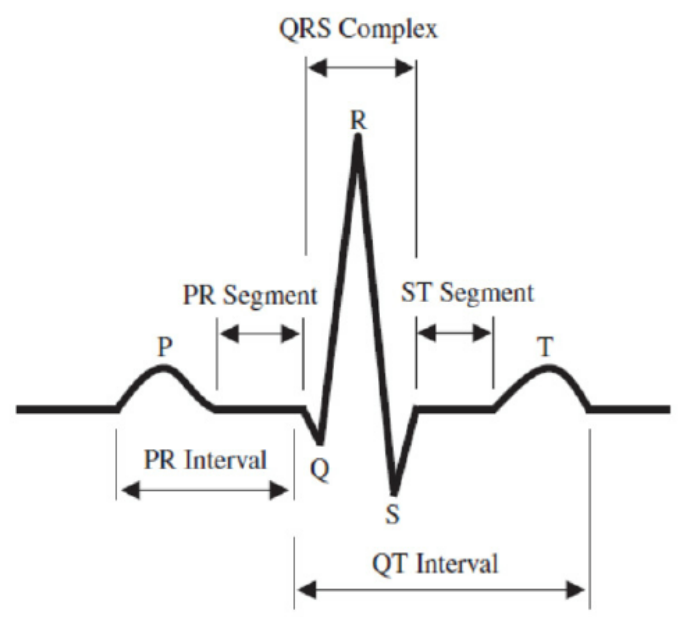

Figure 1: ECG for a single cardiac cycle [26].

According to the medical definition, the most important information from an ECG signal is almost concentrated on the $\mathrm{P}$ wave, QRS complex and $\mathrm{T}$ wave. The QRS complex, because of its very distinguished shape that facilitates its detection, is generally regarded as a reference point for locating the remaining waves [14]. Consequently, one of the crucial steps in real-time computer-based ECG analysis is the accurate detection of the QRS complexes, particularly the $\mathrm{R}$ wave peaks. The detection of the $\mathrm{R}$ wave is easier than other ECG waves due to its structural form and its high amplitude. Currently, it is particularly difficult to achieve QRS complex detection while the monitoring is running during normal daily activity; this is primarily due to morphological differences in the waveform recorded between subjects or due to high noise contamination caused by motion artifacts, muscular movements, or baseline drift changes.

Currently, the unusual pattern detection and prediction of dynamic systems is a topic of scientific interest. The representability by time series is usually used for the observability of real-time monitoring systems. The complexity of heart rate variability signals can be considered as chaotic because of instability and perturbations present during the monitoring; because of this, electrocardiogram (ECG) signal modeling and prediction are difficult. The variations affect the prediction accuracy, and therefore the applications in which ECG signal modeling and prediction have been implemented are compromised. One of the examples that can be mentioned is the application in medical treatment. Because of the above mentioned considerations, ECG monitoring by adaptive models is proposed [12].

In this research work, a pattern monitoring system is constantly fed new data obtained from the heart rate signals acquired from a BITalino biomedical data acquisition card [26]. The system has been implemented by a dynamic quadratic neural unit (DQNU) with sample-by-sample real time recurrent learning (RTRL); in this sense, the system error function decreases considerably and the algorithm is capable of efficiently modeling the cardiac behavior of people from the age of 23 years old in real time. In addition, through the implementation of the adaptive monitoring system, the algorithm is able to recognize patterns that can predict a possible cardiac arrhythmia up to 1 second in advance.

The structure of this research paper is as follows: section 2.1 illustrates different studies related to cardiac arrhythmia detection and prediction; section 2.2 presents a motivating example; the methodology applied for cardiac arrhythmia prediction is described in section 3 ; in section 3.7 , the obtained results are shown and discussed accordingly; section 3.8, presents the comparison of the proposal against related work; finally, conclusions and future work are summarized in section 4 .

\section{Problem Formulation}

\subsection{Related Work}

Next, some works are presented in which a variety of algorithms and tools are used to perform cardiac arrhythmia monitoring. These works were used as references in developing the following research.

Many researchers have addressed the problem of automatically detecting and classifying cardiac rhythms. $\mathrm{K}$-nearest neighbor [15], feedforward neural networks as multilayer perceptron (MLP) [18], support vector machines (SVM) [30], fuzzy or neuro-fuzzy systems [32], Markov models [6] and combinations from different approaches have been used in the literature for this purpose $[7,4,13]$.

Although arrhythmia classification is a challenging problem due to the significant variations in the temporal and morphological characteristics of ECG signals for different patients and under different conditions, several unsupervised learning methods have been developed. [10] applied a new clustering method based on the kernelized fuzzy c-means algorithm to detect arrhythmias by mapping into a higher dimensional space. [34] selected five types of arrhythmias from the MITBIH arrhythmia database to be analyzed, and the diagnosis performance was accurate because of the clustering performance resulting from a new multiclass clustering method based on the maximum margin clustering algorithm and immune evolutionary algorithm. Although there are many ECG clustering algorithms for detecting arrhythmias, these methods present some disadvantages: trapping into local minima, lack of prior knowledge for the parameters of the clustering method, and sensitivity to initialization. In addition to unsupervised methods, supervised methods have also been employed to classify ECG arrhythmias. The SVM method is one of the main supervised learning methods and has been proven to be powerful [30, 7, 13]. As the most well-known method in supervised learn- 
ing, the artificial neural network (ANN) classification method has been extensively applied to ECG arrhythmia classification. In this regard, the MLP has been shown to be able to accurately recognize and classify ECG arrhythmias. In particular, ANN has been integrated with other methods to improve accuracy, such as the use of data reduction and feature extraction techniques, including independent component analysis (ICA) [17], principal component analysis (PCA) [31], wavelet transform [9] and fuzzy logic [22], among others. However, ANN algorithms can become trapped in local minima, and they suffer from random settings of initial values of weights. Additionally, compared to SVM, ANN has less mean square error, but the training stage is slower.

[27] developed an early warning system to monitor cardiac behavior in a person. The information is processed and analyzed to send warning signals to the early warning module in the case of cardiac abnormalities. On the other hand, [23] proposed an algorithm for the detection of normal and abnormal heartbeats from ECG signals, achieving a combined total accuracy rate of $93.44 \%$. [19] proposed a system based on the Internet of Things (IoT) to monitor human biomedical signals in activities that involve physical effort. The information acquired in real time by these devices presented a clear social objective capable of predicting not only situations of sudden death but also possible damage or injuries. [33] proposed a new method for ECG monitoring based on IoT (Internet of Things) techniques. ECG data were gathered using a wearable monitoring node and sent directly to the IoT Cloud using Wi-Fi. Results revealed that the proposed system was reliable for real-time ECG data gathering and display, which can help to discover heart diseases in standard diagnosis.

In contrast to the related work, the presented cardiac arrhythmia prediction in real time consists of monitoring the electrocardiogram signal recorded online from the BITalino biomedical data acquisition card and processing it with the DQNU architecture with RTRL technique. The objective is to predict possible arrhythmia and to detect those arrhythmia patterns.

\subsection{Motivating example}

The works of [8], [5] and [3] have presented QNUs indicating the characteristics of the neural unit for providing a faster response in comparison to a controller employing linear state feedback, as well as to controllers applied to nonlinear systems, unstable systems, and unknown non-linear dynamic systems.

The development of this type of higher-order neural unit activates new perspectives in the solution of complex problems, such as pattern recognition and automatic control: both issues were addressed by [2] and [24]. Benes focused on automatic control, while Rodriguez proposed the use of QNU to reduce the number of neural weights without sacrificing the superior neural unit performance.
The adaptive novelty detection algorithms are addressed with higher-order neural units (HONUs) in [21], where the authors presented two algorithms for adaptive novelty detection based on supervised learning of an HONU and a self-organized map model both with extensions for adaptive monitoring of the existing closed-loops. These new perspectives of HONUs are atributed to the characteristics possessed by the neural units, which contributes to this type of system, which is classified as difficult to model because of high complexity with respect to factors such as flexibility, adaptability, efficiency, and time response, [12].

A higher-order neural unit contains all the nonlinear and linear correlation terms of the input components to the $r$ order. It must be remarked that a generalized structure of a higher-order neural unit is the polynomial network, which includes a weighted sum of products of determined input components with a selected power. A backpropagation algorithm is usually incorporated to train the polynomial networks, and in many cases terms up to second-order are used, while the higher-order terms are not required [25].

In addition, a QNU can be used as a feedforward model for direct prediction of time series with sliding window retraining. The retraining (sliding window) allows capturing the dynamics of the non-stationary time series, and then the prediction becomes closer to the desired real-time target. This method can be considered an offline learning method applied on continuously changing training data. The data organized in the window will be used on a training algorithm, as in batch learning patterns with a single step increment. Backpropagation and the Levenberg-Marquardt learning rule can be used to investigate the performance of sliding window learning and testing with new and different data [25].

To illustrate the performance of QNU with the sliding window technique, the following motivating example is addressed. Here, the data for stock market prediction have been collected for the Standard \& Poor's 500 (S\&P 500) index in the USA. The data consist of technical indicators and monthly closing prices of the index. The total number of samples for the stock index is 676, from 1 January 1957 to 1 April 2013. The data are tested from sample 400 to sample 670 . The inputs are normalized to values between -1 to +1 . The experiments are carried out to test the performance of the models for prediction 3 months in advance. This corresponds to 3 steps ahead, since the sampling occurs every month. The root mean squared error (RMSE) is used to test the performance of the prediction.

The prediction with the static QNU prediction model is shown in Fig. 2. In Figure 2, the $R M S E$ of the 3 steps ahead prediction is 93.0222 of the stock value, with computing time of $10.0881 \mathrm{~s}$. The prediction horizon is obtained for a single length of input vector $\mathbf{x}(k)$ with $n=15$. The number of input-output training samples for the sliding (retraining) window was set as $N_{\text {train }}=100$. 
This example demonstrates the ability of the QNU predictive model for prediction of prices of leading stock indices. In addition, it highlights the interesting characteristics of a sliding window approach for technical applications where the data appear to be highly non-stationary, and it can be concluded that a DQNU can exhibit interesting characteristics for more complicated non-stationary signals, such as ECG, and can achieve superior results due to the ability of the dynamic model to feed back the output of the model for monitoring the predicted output.

\section{Problem Solution}

The proposed system for the automatic adaptive methodology can be observed in the diagram in Figure 3 , where the composition of the general architecture of the methodology is shown.

In the signal acquisition stage, the capture of ECG signals is achieved through patches placed on the surface of the skin on the correct sides of the heart. For the identification of electrodes and their positions, the Bitalino (r)evolution card has been used. BITalino's biomedical development kit is an easy-to-use, lowcost toolkit for learning applications and prototypes using body signals. It consists of a printed circuit board (PCB) with a microcontroller, Bluetooth 2.0, a digital-to-analog converter (DAC), a $3.7 \mathrm{~V}$ to $500 \mathrm{~mA}$ Li-Po battery, an LED actuator, electrocardiography (EGC), electroencephalography (EEG), electromyography (EMG), and electrodermal activity (EDA) sensors, an accelerometer and a light sensor. The small dimensions of this system and the wireless acquisition system allow their implementation in a greater number of experiments [26].

\subsection{ECG signal acquisition}

The data acquisition was performed through the BITalino (r)evolution card, which has all the necessary components to start working with physiological data (see Fig. 4) [26].

\subsubsection{ECG}

Heart beats are activated by very-low-amplitude bioelectric signals generated by a special set of cells in the heart (the SA node). Electrocardiography (ECG) allows the translation of these electrical signals to numerical values, which allows their use in a wide range of applications. This sensor allows the acquisition of data not only in the chest ("in the person"), but also in the palms of the hands ("out of the person"), and it works with pre-gelled electrodes and with most types of dry electrodes. The bipolar configuration is ideal for the acquisition of low-noise data [26].

\subsection{Sampling}

The analog filter processes the analog input to obtain the band-limited signal, which is sent to the analog-todigital conversion unit (ADC). The ADC unit samples the analog signal, quantizes the sampled signal, and encodes the quantized signal levels into the digital signal. An analog signal (continuous time) is defined at each point on the time axis and the amplitude axis. Therefore, the analog signal contains an infinite number of points.

\subsection{Filtering}

A bandpass digital filter is applied to facilitate prediction of the ECG signal. The applied bandpass filter is called a Butterworth filter. The frequency response in the passband and in the stopband is as flat as possible; therefore, this filter is also called the maximally flat magnitude filter. This filter has a minimum phase change over the passband compared to other conventional IIR filters. On the other hand, the output decreases at a rate of $-20 \mathrm{~dB}$ per decade per pole, and the phase response becomes less linear with increasing order of the filter. Compared to Chebyshev and elliptical filters, the Butterworth filter has a slower output signal but a more linear phase response in the passband.

\subsection{Training process}

The DQNU uses an RTRL learning method and can be implemented in discrete or continuous real time. The notation of DQNU with RTRL is shown in Eq. (1), where $\widetilde{y}(k+h)$ is the predicted neural output and ${ }^{T}$ represents the transpose of the vector $\mathbf{x}(k)$, which contains the feedback neural output values and the signal from the heart rate variability (HRV) [12].

$$
\widetilde{y}(k+h)=\left[\sum_{i=0}^{n x-1} \sum_{j=0}^{n x-1} x_{j} \cdot w_{i, j} \cdot x_{i}\right]
$$

The upper triangular matrix of weights $\mathbf{W}$ with neural bias $w_{0,0}$ is defined in Eq. (2).

$$
\mathbf{W}=\left[\begin{array}{cccc}
w_{0,0} & w_{0,1} & \ldots & w_{0, n x-1} \\
0 & w_{1,1} & \cdots & w_{1, n x-1} \\
\vdots & \vdots & \ddots & \vdots \\
0 & \cdots & 0 & w_{n x-1, n x-1}
\end{array}\right]
$$

Equation (3) is the column of the augmented vector $\mathbf{x}(k)$ of the neural input, $\mathbf{y}$ stands for the real values, $n$ is the number of real values that feed the neural input, $k$ is the variable that describes the discrete time and $h$ is the prediction horizon. 
Prediction by QNU

RMSE $=93.0222$, computing time $=10.0881 \mathrm{~s}$

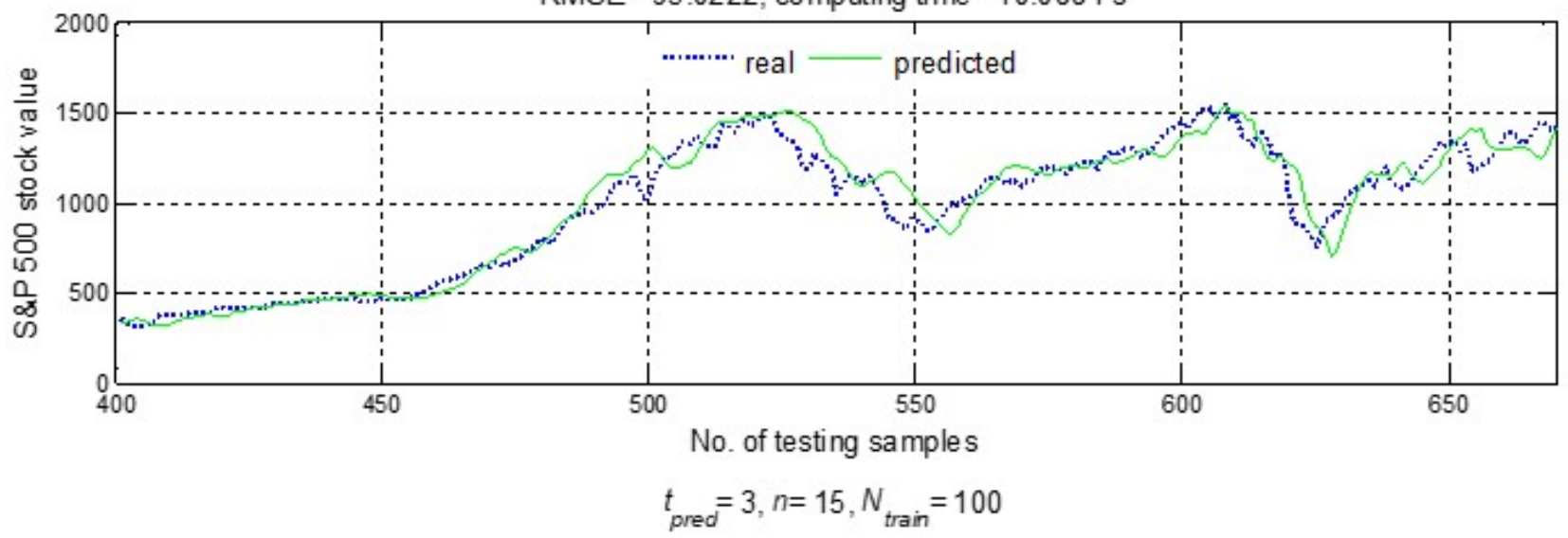

Figure 2: Prediction of S\&P 500 data by the QNU prediction model with the sliding window approach.The prediction is 3 steps ahead.
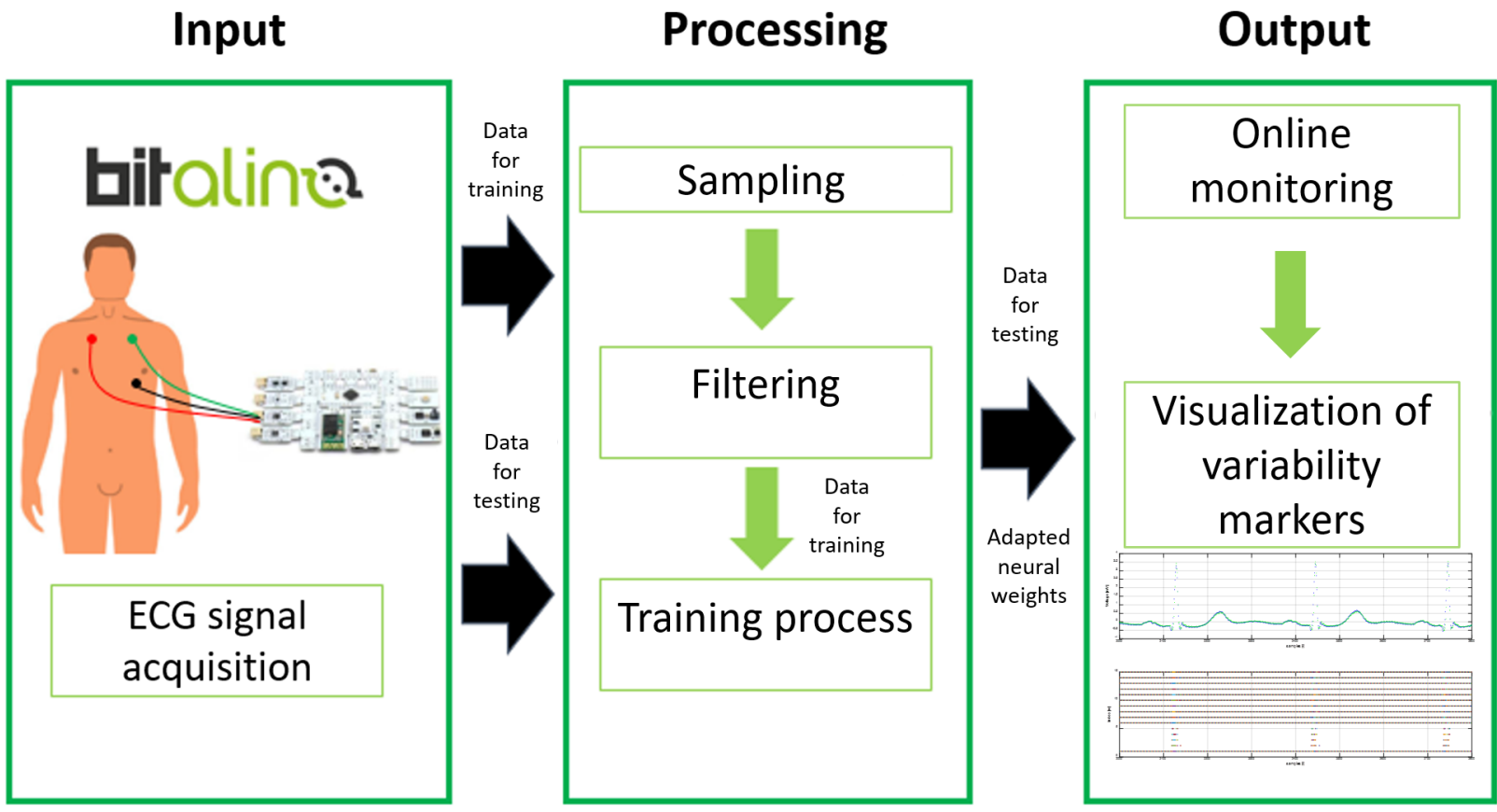

Figure 3: Proposed adaptive methodology for the monitoring system.

$$
\mathbf{x}(k)=\left[\begin{array}{c}
1 \\
\tilde{y}(k+h-1) \\
\tilde{y}(k+h-2) \\
\vdots \\
\tilde{y}(k+1) \\
y(k) \\
y(k-1) \\
\vdots \\
y(k-n+1)
\end{array}\right]
$$

In Eq. (4), $w_{i, j}(k+1)$ stands for the adaptation of neural weights in the new adaptation time of the model: the sum of the neural weights increment $\Delta w_{i, j}(k)$ from the adaptation of the model and individual weights $w_{i, j}(k)$ of each discretized value from the HRV.

$$
w_{i, j}(k+1)=w_{i, j}(k)+\Delta w_{i, j}(k)
$$

The increment of the neural weights with RTRL is described in Eq. 5, where $\mu$ is the learning rate that determines in which proportion the neural weights are updated and the velocity of the learning process, $e(k+h)$, 


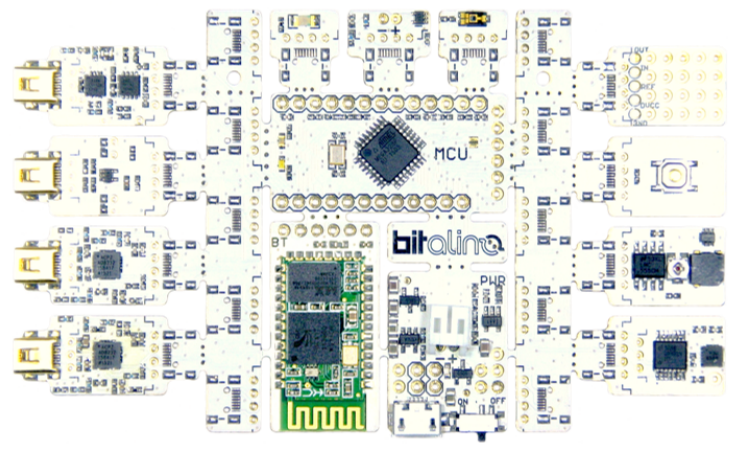

Figure 4: BITalino (r)evolution card [26].

is the error in each discrete time $(k+h)$ that multiplies the partial derivative of the neural output with respect to the weights $w_{i, j}$.

$$
\Delta w_{i, j}(k)=\mu \cdot e(k+h) \cdot \frac{\partial \tilde{y}(k+h)}{\partial w_{i, j}}
$$

\subsection{Online monitoring}

The DQNU proposed model is under research, and the development of this methodology has been shown to be useful in real-time evaluation processes of dynamic systems from real-world and theoretical systems of chaotic behavior.

The DQNU system contains an adaptive graphic monitor which is used for displaying the HRV in real time. This system consists of detecting and visualizing unusual patterns that can indicate possible cardiac arrhythmias in patients, by adapting the neural weights during the model adaptation. In the system, sampleby-sample adaptation was implemented based on the gradient descent rule with RTRL [12].

The predictive model monitors the online records of electrocardiograms (ECG) obtained from the BITalino biomedical data acquisition card. This makes the predictive model able to identify patterns in time series and to achieve the prediction of values of HRV during the real-time monitoring. This monitoring is performed on a different data set utilized for the training stage, which is illustrated on Fig. 3.

\subsection{Visualization of variability markers}

In the adaptive methodology, the neural weights are initially random values from -1 to 1 , and such values are updated by the learning process in every iteration time, with respect to the system training intervals. During this process, the sum of squared error is reduced to the vicinity of the global minimum. The predictive model acquires data continuously by sample-by-sample adaptation. Unusual adaptive neural weights are evaluated and visualized by a graphical interface design. For the detection of changes in the signal, a sensitivity parameter is initialized experimentally as $\alpha=2.95$. This sensitivity parameter is considered in the online monitoring in Fig. 3. Online monitoring influences a condition that allows drawing markers according to the evaluation of the increment parameters. To allow drawing of the markers, a condition is considered as in Eq. 6.

$$
|\Delta \mathbf{W}(k)| \geq \alpha \cdot \overline{\Delta \mathbf{W}(k)}
$$

where $|\cdot|$ stands for the absolute value, and $\overline{\Delta \mathbf{W}(k)}$ corresponds to the average of the weight increments. Accordingly, a window of a set of values has been considered for the average weight increment.

This adaptation of DQNU converges singularly and rapidly to the vicinity of the global minimum thanks to the gradient descent methodology. The configurations of inputs and outputs of the DQNU are nonlinear; however, the optimization of the neural weights is a linearly solvable task due to the DQNU mathematical structure. It is important to point out that the mathematical structure does not present problems with local minima due to the adjustment made by the quality of the training data; however, improper training data will significantly affect the predictive model [12].

\subsection{Experimental results}

The recorded data were utilized to validate the adaptive monitoring system proposed in this article. The arrhythmia database contains sampled records for the adaptive monitoring. Records contain beats, which are duly identified during the real-time adaptive monitoring. It is important to point out that the adaptive model is capable of identifying variabilities of ECG arrhythmias, showing different patterns for each case.

The DQNU has been presented in section 3 for predicting ECG signals. The input configuration of the model was $(n+h)$, where $n$ stands for the number of samples of the signal used to feed the model and $(h-1)$ is the number of feedbacks to feed the input model. The bias $\mathbf{x}(k=1)=1$. The number of training times has been set to epochs $=50$, and the learning rate has been configured to $\mu=0.0001$. In addition, a window for the average of the neural weights increments has 
been considered as window $=180$, the initial weights of the adaptive model have been initialized as random normalized values. Also, the sensitivity parameter has been set to $\alpha=2.95$, which will be used in the visualization of the variability markers.

Because the adaptive model is based on RTRL, sample-by-sample adaptation, it can be observed that the neural output predicts the behavior of the unstable signal of the real cardiac signals, while markers indicate the relevant changes of the sampled signal, allowing the patterns formed in each type of cardiac arrhythmia to be displayed.

Fig. 5 shows the results of the adaptive monitoring of a patient with Premature Ventricular Contraction (PVC) arrhythmias. In the upper subplot, the real signal is shown with blue lines, and the predicted signal in real-time monitoring is superimposed with green lines. Also, the lower subplot presents the visualization of patterns of the variability markers obtained for the PVC arrhythmia. PVC arrhythmias are highlighted in the vertical red blocks.

Fig. 6 presents the results of the adaptive monitoring of the ECG obtained from a patient with Atrial Premature Contraction (APC) arrhythmia. In the upper subplot, the real signal is presented with blue line color, and the predicted signal in real-time monitoring is shown by green lines. In addition, the lower subplot shows the variability patterns visualization with the markers obtained from the adaptive monitoring system. The APC arrhythmia is highlighted in the vertical red blocks in Fig. 6.

Fig. 7 presents the results of the adaptive monitoring of the ECG obtained from a patient with normal hertbeat variability. In the upper subplot, the real signal is presented with blue lines, and the predicted signal in real-time monitoring is shown by green lines. In addition, the lower subplot shows the visualization of variability patterns with the markers obtained from the adaptive monitoring system. The normal heartbeats are highlighted in the vertical red blocks in Fig. 7 .

\subsection{Comparison with related works}

The main contribution of this research is the prediction and monitoring of the HRV in order to provide a tool to facilitate medical diagnosis of various cardiac abnormalities in patients and therefore avoid premature death because of an improper medical diagnosis.

Because of the above, several records were utilized in the adaptive monitoring system for validation, including heartbeat records with inverse polarity, low amplitude, ventricular ectopics with low signal noise-toratio, premature ventricular heartbeats and premature atrial heartbeats. In Fig. 5, Fig. 6, and Fig. 7 it can be demonstrated that the presented methodology meets the objective of identifying patterns associated with the different types of cardiac abnormalities during real-time monitoring as well as identifying patterns related to the variability of normal heartbeats. In ad- dition, a different data set was used during real-time monitoring versus those used during the training phase of the system in order to monitor different data from those learned by the model. The variability markers are shown through the adaptive monitoring graphics which indicate the proper detection of the different patterns that might arise during the real-time monitoring.

The main advantage of the methodology of the DQNU model with respect to other methodology types addressed by various authors is that a second-order correlation with the RTRL method is applied to capture nonlinear and complex behavior of the HRV. In addition, the mathematical notation remains relatively simple in comparison with other methodologies because their complexity requires greater processing. Besides, DQNU does not present problems with local minima during its optimization.

Currently, an automatic pattern recognition system is required, which enables automatic classification of each cardiac abnormality shown above. In addition, cloud service will be enabled through a connection between a server and raspberry PI as data acquisition card. With this cloud service, the cardiac arrhythmia pattern detection of the proposed model will be implemented in medical institutions.

\section{Conclusion}

The proposed system is currently in the experimental stage; however, the results of the monitoring system for prediction of cardiac arrhythmias allow us to conclude according to the figures shown in section 3.7, that the adaptive model predicts the behavior of the HRV and presents the variability behavior on a monitor plot. The model of recurrent learning in real time has been proven capable of providing the system with the necessary neural weights adaptation on which the values prediction of HRV is based. This, is attributed to the algorithm of backpropagation of the error in sampleby-sample adaptation during training. In its current stage, the adaptive methodology process plots the variability markers, and the patterns of variability are visualized.

Future work will focus on increasing the prediction horizon without sacrificing the accuracy of the adaptive model. In addition, a pattern recognition method will be included in the monitoring system, and automatic recognition of different types of arrhythmias that may occur during signal monitoring will also be included.

Acknowledgement: This project is supported by Jan Evangelista Purkyně University. Title of the project: Predictive maintenance of an industrial machine using neural networks. 


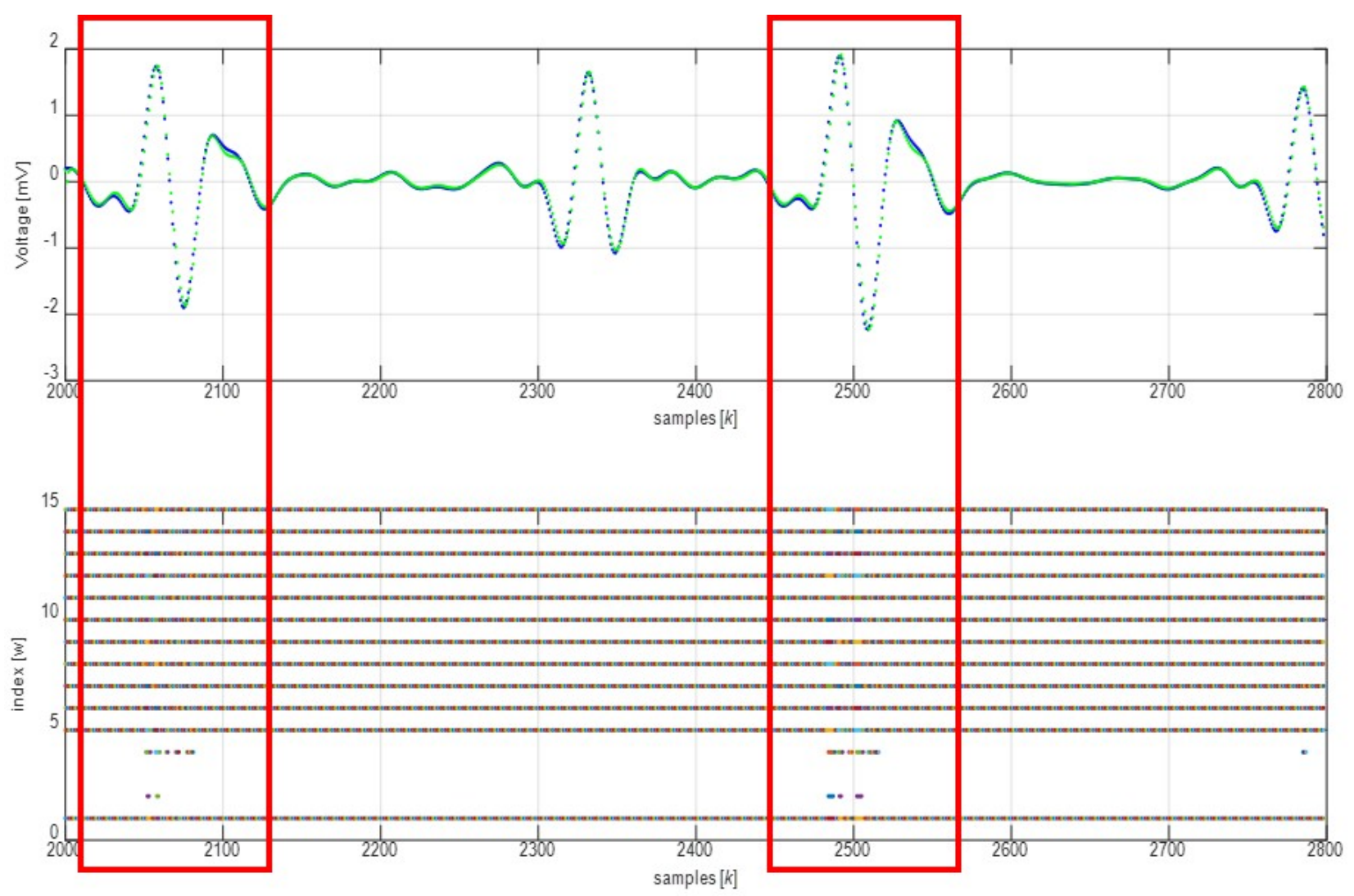

Figure 5: Adaptive monitoring for the detection of PVC arrhythmias.

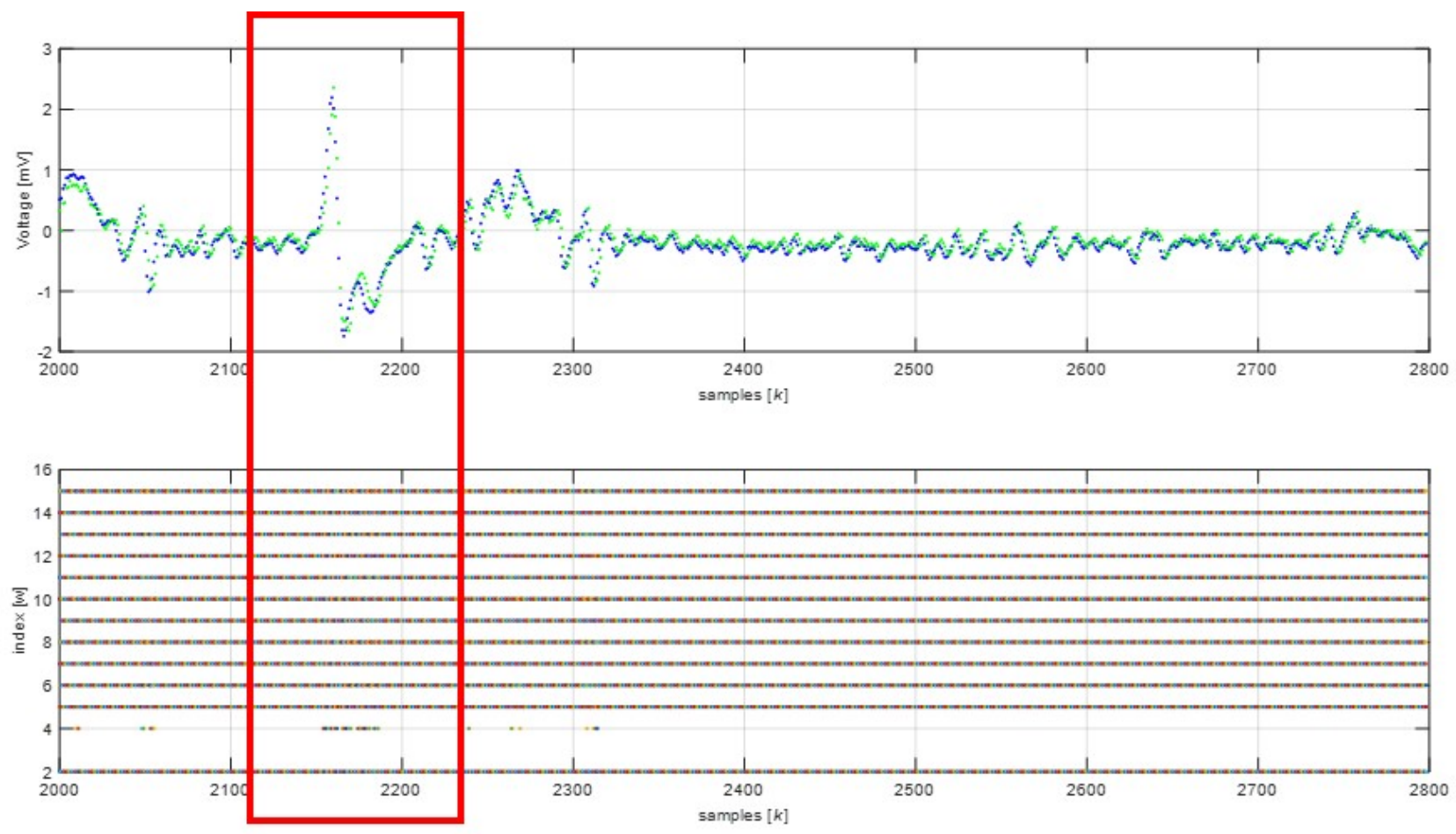

Figure 6: Adaptive monitoring for the detection of APC arrhythmia. 


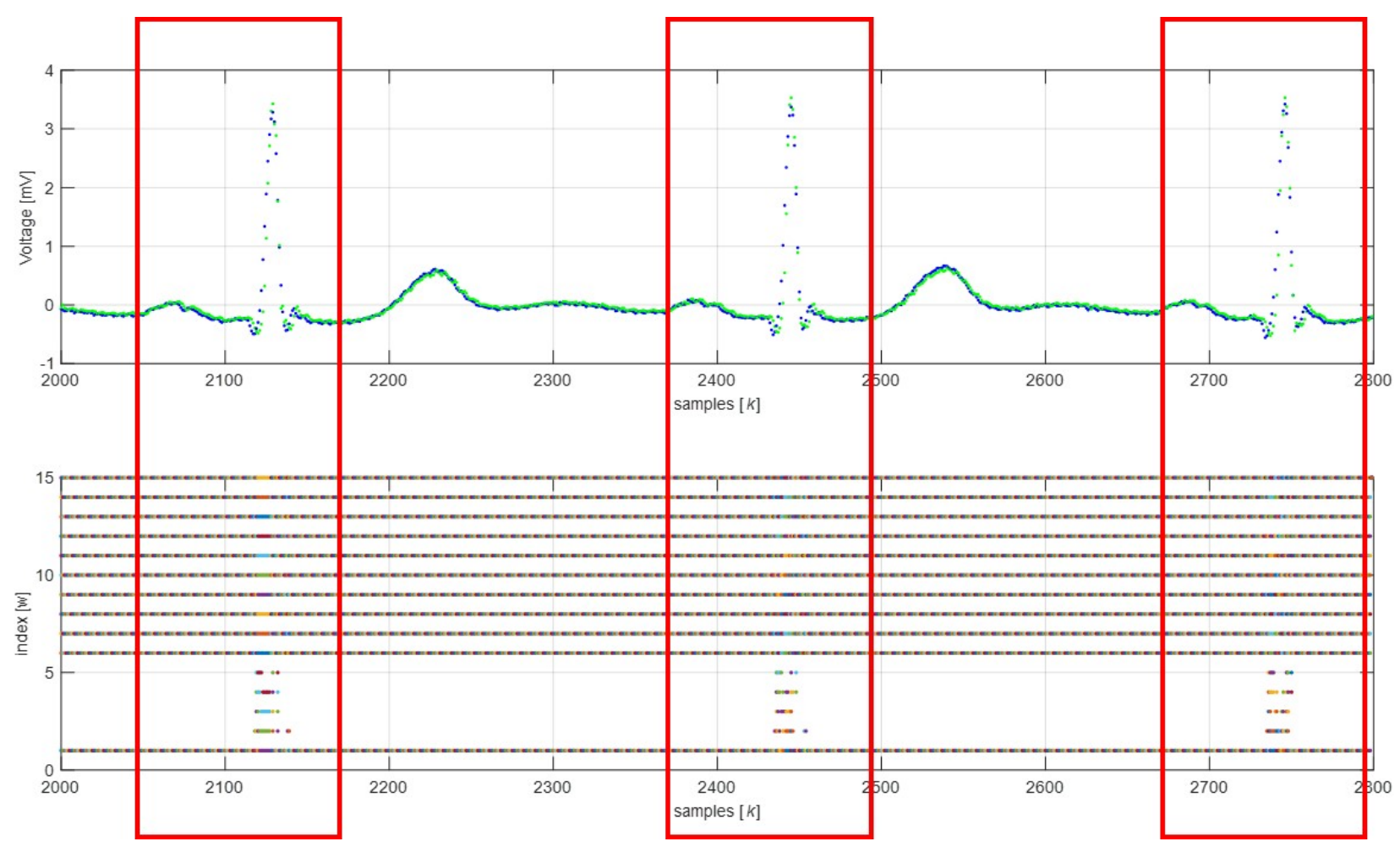

Figure 7: Adaptive monitoring for the detection of normal heartbeat.

\section{References}

[1] Afrhami, R., Azarnia, G., and Ali, M. Cardiac arrhythmia classification using statistical and mixture modeling features of ecg signals. Pattern Recognition Letters 70 (2016), 45-51.

[2] Benes, P. M., Erben, M., Vesely, M., Liska, O., AND Bukovsky, I. Honu and supervised learning algorithms in adaptive feedback control. Applied Artificial Higher Order Neural Networks for Control and Recognition (2016), $35-60$.

[3] Bukovsky, I., Homma, N., IchiJi, K., CeJnek, M., Slama, M., Benes, P. M., And BILA, J. A fast neural network approach to predict lung tumor motion during respiration for radiation therapy applications. BioMed research international 2015 (2015), 1 - 13.

[4] Castillo, O., Melin, P., Ramírez, E., And SORIA, J. Hybrid intelligent system for cardiac arrhythmia classification with fuzzy k-nearest neighbors and neural networks combined with fuzzy system. Expert Syst. Appl. 39, 3 (2012), 29472955.

[5] Cejnek, M., Bukovsky, I., Homma, N., And LiskA, O. Adaptive polynomial filters with individual learning rates for computationally efficient lung tumor motion prediction. International Workshop on Computational Intelligence for Multimedia Understanding (2015), $1-5$.

[6] Chang, P., Lin, J., Hsien, J., and Weng, J. Myocardial infarction classification with multi- lead ecg using hidden markov models and gaussian mixture models. Appl. Soft Comput. 12, 10 (2012), 3165-3175.

[7] Chen, H., Cheng, B., Liao, G., and Kuo, T. Hybrid classification engine for cardiac arrhythmia cloud service in elderly healthcare management. J. Visual Lang. Comput. 25, 6 (2014), 745753.

[8] Cordero, R., Suemitsu, W., and Pinto, J. Analysis and convergence theorem of complex quadratic form as decision boundary for data classification. Electronics Letters 51, 7 (2015), 561562.

[9] Dahmouche, A., Hamami, L., Alajlan, N., And Melgani, F. A wavelet optimization approach for ecg signal classification. Biomed. Signal Process. Control 7, 4 (2012), 342-349.

[10] Dogan, B., And Korurek, M. A new ecg beat clustering method based on kernelized fuzzy c-means and hybrid ant colony optimization for continuous domains. Appl. Soft Comput. 12, 11 (2012), 3442-3451.

[11] Elgendi, M., Eskofier, B., Dokos, S., And Аввотт, D. Revisiting qrs detection methodologies for portable, wearable, battery-operated, and wireless ecg systems. PLOS ONE 9, 1 (2014), 118.

[12] Herrera, J., Rodriguez-Jorge, R., Villegas, O., Cruz, V., Bila, J., Nandayapa, M. J., Ponce, I., Soto, A. I., And Flores, A. Monitoring of cardiac arrhythmia patterns 
by adaptive analysis. Advances on P2P, Parallel, Grid, Cloud and Internet Computing. 3PGCIC 2016. Lecture Notes on Data Engineering and Communications Technologies 1 (2017), 885-894.

[13] Homaeinezhad, M., Atyabi, S., Tavakkoli, E., Toosi, H., Ghaffari, A., And EbrahimPOUR, R. Ecg arrhythmia recognition via a neurosvm-knn hybrid classifier with virtual qrs imagebased geometrical features. Expert Syst. Appl. 39, 2 (2012), 2047-2058.

[14] Karimipour, M., And Homaeinezhad, M. R. Real-time electrocardiogram p-qrs-t detectiondelineation algorithm based on quality-supported analysis of characteristic templates. Computers in Biology and Medicine 52 (2014), 153 - 165.

[15] Kutlu, Y., And KuntalP, D. A multi-stage automatic arrhythmia recognition and classification system. Comput. Biol. Med. 41, 1 (2011), 37-45.

[16] Luz, E., Schwartz, W., CÁmara-Chávez, G., And MenotTi, D. Ecg-based heartbeat classification for arrhythmia detection: A survey. Computer Methods and Programs in Biomedicine 127 (2016), 144-164.

[17] Martis, B., Acharya, U., Prasad, H., Chua, C., Lim, C., And Suri, J. Application of higher order statistics for atrial arrhythmia classification. Biomed. Signal Process. Control 8 (2013), 888900.

[18] Mohan, H., Trivedi, A., And Shukla, S. Ecg signal processing for abnormalities detection using multi-resolution wavelet transform and neural network classifier. Measurement 46, 9 (2013), 3238-3246.

[19] Mora, H., Gil, D., Terol, R. M., Azorn, J., AND SzYMANSKI, J. An iot-based computational framework for healthcare monitoring in mobile environments. Sensors 17, 10 (2017), 2302.

[20] Organization, W. H. Noncommunicable diseases: progress monitor 2020. World Health Organization, 2020.

[21] Oswald, C., Cejnek, M., VRBa, J., AND Bukovsky, I. Novelty detection in system monitoring and control with honu. Applied Artificial Higher Order Neural Networks for Control and Recognition (2016), 61-78.

[22] Ozbay, Y., Ceylan, R., and Karlik, B. Integration of type-2 fuzzy clustering and wavelet transform neural network based ecg classifier. Expert Syst. Appl. 38, 1 (2011), 1004-1010.

[23] Pandit, D., Zhang, L., Liu, C., ChattopadHyay, S., Aslam, N., AND Lim, C. A lightweight qrs detector for single lead ecg signals using a maxmin difference algorithm. Computer Methods and Programs in Biomedicine 144 (2017), 61-75.

[24] Rodriguez, R., Bukovsky, I., And Homma, N. Potentials of quadratic neural unit for applications. Advances in Abstract Intelligence and Soft Computing (2013), 343-354.
[25] Rodriguez-Jorge, R. Artificial neural networks: Challenges in science and engineering applications. Frontiers in Artificial Intelligence and Applications 295 (2017), 25--35.

[26] Rodríguez-Jorge, R., León-Damas, I. D., AND BILA, J. Detection of the qrs complexity in real time with bluetooth communication. Advances on P2P, Parallel, Grid, Cloud and Internet Computing. 3PGCIC 2020. Lecture Notes in Networks and Systems 158 (2021), 429-439.

[27] Sahoo, P. K., Thakkar, H. K., And LeE, M. Y. A cardiac early warning system with multi channel scg and ecg monitoring for mobile health. Sensors (Basel) 17, 4 (2017), 711.

[28] Salles, G., Cardoso, C., Fonseca, L., FiszMAN, R., AND Muxfeldt, E. Prognostic significance of baseline heart rate and its interaction with beta-blocker use in resistant hypertension: a cohort study. Am. J. Hypertens 26, 2 (2013), 218 226.

[29] Shadmand, S., And Mashouf, B. A new personalized ecg signal classification algorithm using block-based neural network and particle swarm optimization. Biomedical Signal Processing and Control 25 (2016), 12-23.

[30] Sun, S. An innovative intelligent system based on automatic diagnostic feature extraction for diagnosing heart disease. Knowl-Based Syst. 75 (2015), 224-238.

[31] Wang, J., Chiang, W., Hsu, Y., And Yang, Y. Ecg arrhythmia classification using a probabilistic neural network with a feature reduction method. Neurocomputing 116 (2013), 38-45.

[32] WEE, T., AND WAN, W. Non-singleton genetic fuzzy logic system for arrhythmias classification. Eng. Appl. Artif. Intell. 24, 2 (2011), 251-259.

[33] Yang, Z., Zhou, Q., Lei, L., Zheng, K., And XiAnG, W. An iot-cloud based wearable ecg monitoring system for smart healthcare. Journal of medical systems 40, 12 (2016), $286-723$.

[34] Zhu, B., Ding, Y., And HaO, K. Multiclass maximum margin clustering via immune evolutionary algorithm for automatic diagnosis of electrocardiogram arrhythmia. Appl. Math Comput. 227 (2014), 428-436. 